\title{
Improvement in Pulmonary Function After Closure of Atrial Septal Defect in a Patient With Cystic Fibrosis
}

\author{
Abdulfattah Saidi, $\mathrm{MD}^{1 *}$, Holly Carveth, $\mathrm{MD}^{2}$, Anwar Tandar, MD ${ }^{1}$ \\ ${ }^{1}$ Division of Cardiovascular Medicine, University of Utah Hospital, Salt Lake City, Utah, United States \\ ${ }^{2}$ Division of Pulmonary Medicine, University of Utah Hospital, Salt Lake City, Utah, United States
}

\begin{abstract}
Atrial septal defect (ASD) is a major cause of left-toright intracardiac shunting. If persisting into adulthood, an ASD can lead to a larger shunt, which may eventually cause pulmonary hypertension and right ventricular failure. Large intracardiac shunts cannot be tolerated in patients with underlying lung disease such as cystic fibrosis. Although the association between an intracardiac shunt and cystic fibrosis has been reported in the literature, the impact of ASD closure on the clinical course of patients with cystic fibrosis has not been studied. Here, we report a case of ASD closure in a patient with cystic fibrosis with hypoxemia out of proportion to his lung disease. Closure of the ASD shunt resulted in significant improvement of his symptoms and pulmonary function testing.

Copyright (c) 2017 Science International Corp.
\end{abstract}

\section{Key Words}

Atrial septal defect - Septal closure device - Cystic fibrosis

\section{Introduction}

Secundum atrial septal defect (ASD) is an isolated defect in the fossa ovalis between the atria. Although many ASDs close spontaneously during the first year after birth [1], the defect persists in some cases, causing a left-to-right shunt [2]. Left-to-right shunting,

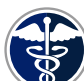

Fax +1 2037853346

E-Mail: jshd@scienceinternational.org

http://structuralheartdisease.org/

\author{
(c) 2017 Journal of Structural Heart Disease \\ Published by Science International Corp. \\ ISSN 2326-4004 \\ Accessible online at: \\ http://structuralheartdisease.org/
}

when significant, can cause dyspnea on exertion, which may not be well tolerated in patients with an underlying pulmonary condition such as cystic fibrosis. It is unknown whether ASD closure could lead to symptomatic improvement in these patients. Here, we report a case of ASD closure resulting in improved pulmonary function testing in a patient with cystic fibrosis with a bidirectional shunt and worsening functional capacity.

\section{Case Presentation}

Our patient was a 36-year-old man with cystic fibrosis diagnosed at birth with a dF508/dF508 genotype. His symptoms could be characterized as moderate airway disease complicated by chronic airway infections, pancreatic insufficiency, diabetes, and malnutrition. Progression of his clinical condition caused dyspnea with minimal exertion. He was referred to our cardiology service to be evaluated for hypoxemia out of proportion to his chronic pulmonary disease. Pulmonary function testing showed a forced expiratory volume in $1 \mathrm{~s}$ (FEV1) of 1.71 (43\% predicted). Transesophageal echocardiography (TEE) showed a small ASD with a bidirectional shunt seen at rest (Figure 1). Right heart catheterization showed a pulmonary artery pressure of $26 / 7 / 16 \mathrm{mmHg}$ and pulmonary vascular resistance of 1.7 Wood units. A decision was made to proceed with ASD closure, which

* Corresponding Author:

Abdulfattah Saidi, MD

Division of Cardiovascular Medicine

University of Utah Hospital

50 North Medical Drive, Salt Lake City, UT 84132, USA

Tel. +801 585 5559; Fax: +801 581 7735; E-Mail: abdulfattah.saidi@hsc.utah.edu 


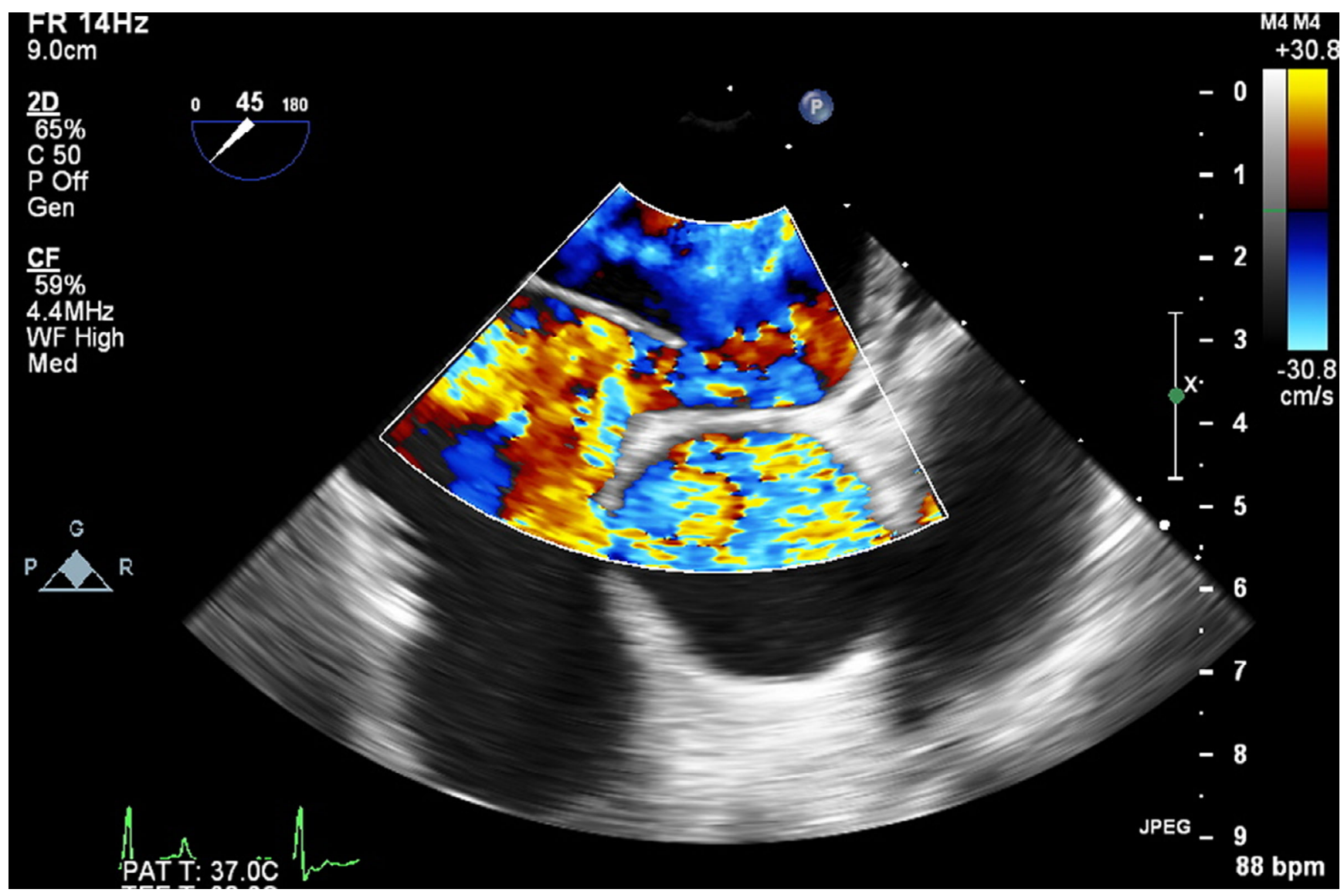

Figure 1. TEE showing bidirectional shunt before 20-mm Occluder Septal Helex placement.

was performed under the guidance of intra-cardiac echocardiography and fluoroscopy. A balloon sizing the defect was used, and the patient underwent ASD closure with a 25-mm Occluder Septal Helex. The indication for this closure was the presence of bidirectional flow that would ultimately lead to irreversible pulmonary hypertension. At the end of the procedure, an agitated saline study was performed, which revealed minimal residual shunt. Echocardiography performed the next day showed very minimal flow across the device as demonstrated by color Doppler. The patient was discharged to home on $81 \mathrm{mg}$ aspirin and $75 \mathrm{mg}$ clopidogrel daily for 3 months. He initially demonstrated symptomatic improvement with less oxygen requirement at rest. However, 3 months later, his symptoms of fatigue and dyspnea on exertion returned, and follow-up echocardiography demonstrated a large right-to-left intra-cardiac shunt at rest as demonstrated by an agitated saline study
(Figure 2). His pulmonary function testing during this time showed an FEV1 of 1.78 (47\% predicted). The patient was monitored for 1 year, during which his functional capacity and cystic fibrosis symptoms remained stable. He subsequently suffered from a neurological event suggestive of a transient ischemic attack of an embolic nature, after which he was considered for residual shunt closure. TEE revealed a deformity of the Helex device causing a bidirectional shunt via the inferior rim of the device. With further questioning, the patient admitted to using a high-frequency chest wall oscillation device a few days after his ASD closure as part of his routine treatment for cystic fibrosis.

The patient underwent closure of the residual shunt using a 30-mm Cardioform device. The second device was advanced across the defect and positioned to sandwich both sides of the previously placed Helex Occluder (Figure 3). The absence of a 


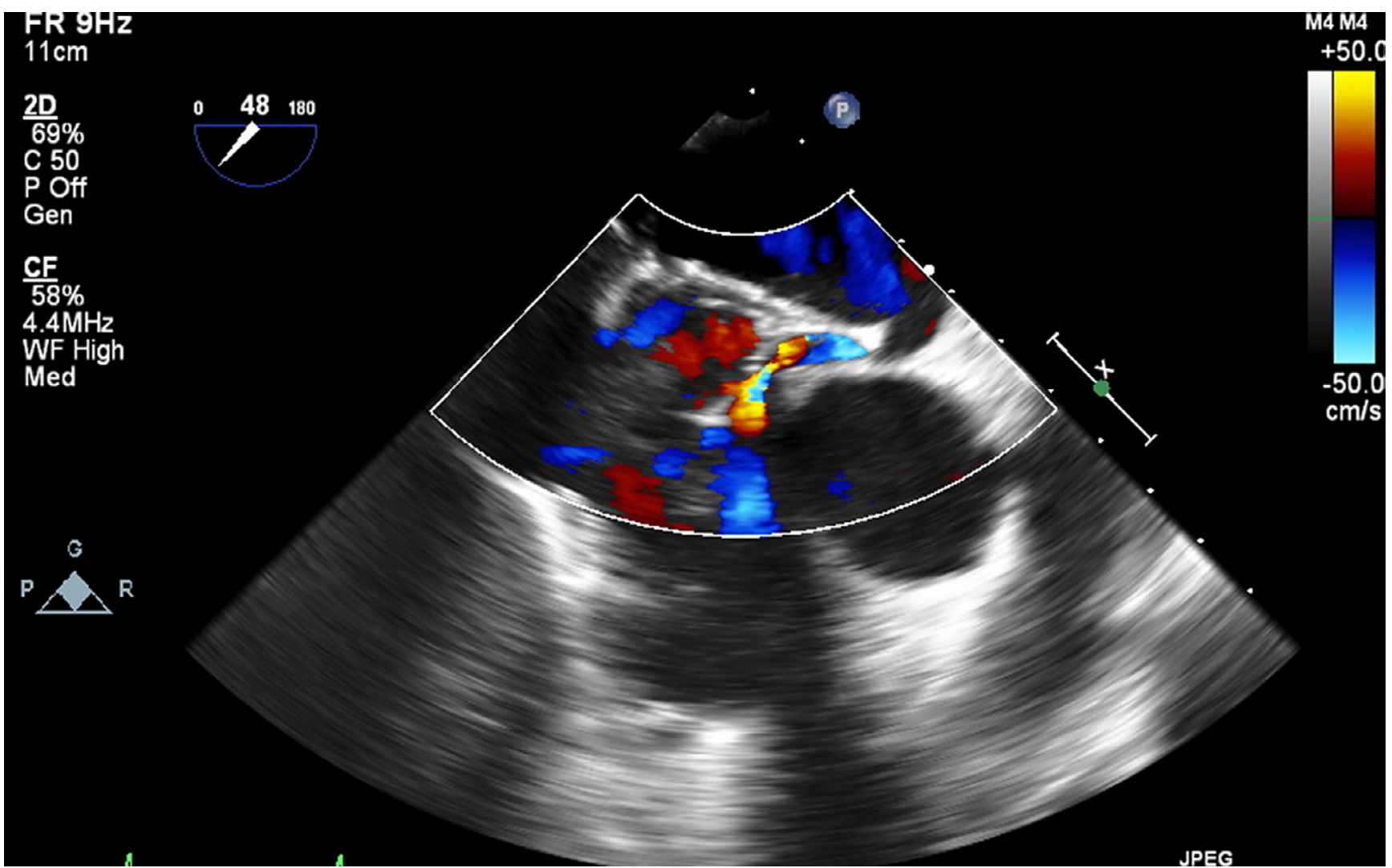

Figure 2. TEE showing residual shunt 1 year after 20-mm Occluder Septal Helex implantation.

residual shunt was confirmed with several negative agitated saline injections. He was advised to not use the high-frequency chest wall oscillation device for at least 12 months. At 6-month follow-up, the patient showed significant improvement in his functional capacity, with pulmonary function testing demonstrating a spike increase in FEV1 to 3.4 (89\% predicted; Table 1).

\section{Discussion}

ASD is one of the most common adult congenital heart defects $[3,4]$. It is caused by underdevelopment of the secundum septum or over-reabsorption of the primum septum. In most cases, ASDs close spontaneously during infancy [4,5]; however, if persistent, their clinical impact is related to their location, size, and association with other defects $[5,6]$. Small ASDs usually cause a left-to-right shunt without significant structural consequences in the right-sided heart chambers. However, larger ASDs, if not corrected in

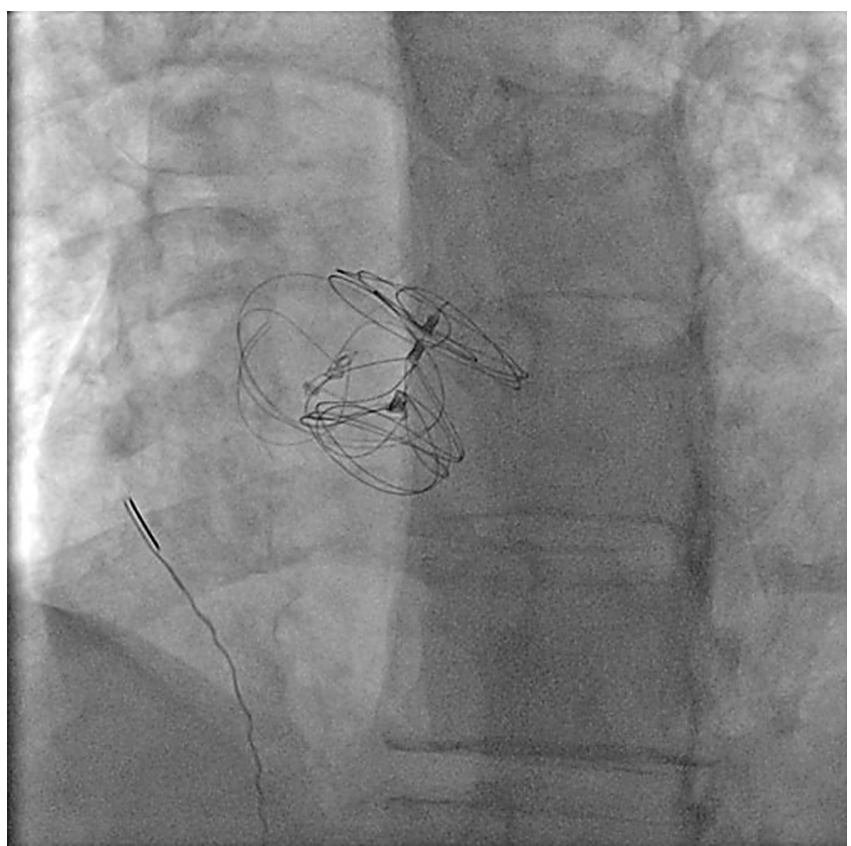

Figure 3. Fluoroscopy showing placement of a 30-mm Cardioform device adjacent to the previously placed 20-mm Occluder Septal Helex. 
Table 1. Pulmonary function test of the patient over his clinical course.

\begin{tabular}{llll}
\hline & Before first ASD closure & After first ASD closure (with residual shunt) & $\begin{array}{l}\text { After second ASD closure (no } \\
\text { significant residual shunt) }\end{array}$ \\
\hline FEV1 & $1.71(43 \%$ predicted $)$ & $1.78(47 \%$ predicted $)$ & $3.4(89 \%$ predicted $)$ \\
FEV1/FVC & $53(65 \%$ predicted) & $61(76 \%$ predicted $)$ & $76(95 \%$ predicted) \\
\hline
\end{tabular}

early life, may lead to right-sided volume followed by pressure overload [6, 7], resulting in right atrial and ventricle enlargement and ultimately pulmonary vasculature remodeling and pulmonary hypertension with symptoms of right heart failure such as fatigue, decrease in functional capacity, and lung infections $[7,8]$. The association between ASDs and cystic fibrosis is not well established. Two small series of patients with cystic fibrosis reported a 53-55\% incidence of left-to-right shunt, mainly due to the presence of a patent foramen ovale (PFO) as seen by TEE $[8,9]$. We can assume that patients with severe lung disease, such as cystic fibrosis in our case, will not tolerate hypoxemia caused by a significant right-to-left shunt. Current American College of Cardiology/American Heart Association guidelines [10] recommend percutaneous interventional therapy for ASDs in the presence of right atrial and right ventricle enlargement with or without symptoms before progressing to irreversible pulmonary hypertension, the presence of which is considered a contraindication for ASD closure. The impact of ASD closure on pulmonary function testing in patients with cystic fibrosis is not known. Belton et al. [11] reported a 29-year-old man with cystic fibrosis who underwent PFO closure after developing neurological symptoms. His pulmonary function had deteriorated at the time of the neurological event. Closure of his PFO improved his pulmonary function with an increase in FEV1 from 1.36 to 1.89 ( $33 \%$ to $47 \%$ predicted). The authors concluded that closure of the PFO may have had a significant impact on lung function. The indication for the first ASD closure in our case was the presence of bidirectional flow, for which we aimed to prevent the development of irreversible pulmonary hypertension that would preclude ASD closure. The first closure significantly improved pulmonary function testing before the device migrated and led to a residual shunt with worsening symptoms and test results. We assume that the use of a high-frequency chest wall oscillation device dislodged the initial occlude, resulting in larger residual shunt. The indication for the second closure was a neurological event caused by a paradoxical embolism. There was significant improvement in pulmonary function testing after the second ASD closure, which was not associated with a residual shunt immediately after the procedure.

In conclusion, percutaneous closure of ASD in patients with cystic fibrosis and hypoxemia out of proportion to their lung disease may improve pulmonary function testing and hypoxemia, although further studies are warranted to confirm this possibility.

\section{Conflict of Interest}

The authors have no conflict of interest relevant to this publication.

\section{Comment on this Article or Ask a Question}

\section{References}

1. Hartmann AF, Elliott LP. Spontaneous physiological closure of atrial septal defect after infancy. Am J Cardiol. 1967;19:290-292. DOI: 10.1016/0002-9149(67)90549-8

2. Brassard M, Fouron JC, Van Doesburg NH, Mercier L, De Guise P. Outcome of children with atrial septal defect considered too small for surgical closure. Am J Cardiol. 1999;83:1552-1555. PMID: 10363870
3. Radzik D, Davignon A, Von Doesburg N, Fournier A, Marchand T, Ducharme G. Predictive factors for spontaneous closure of atrial septal defects diagnosed in the first 3 months of life. J Am Coll Cardiol. 1993;22:851-853. PMID: 8354823

4. Mcmahon CJ, Feltes TF, Fraley JK, Bricker JT, Gifka RG, Tortoriello TA, et al. Natural history of growth of secundum atrial septal defects and implications for transcatheter closure. Heart. 2002;87:256-259. PMID: 11847166

5. Hansilk A, Pospisil U, Salzer-Muhar U, Greber-Platzer S, Male C. Predictors of spontaneous closure of isolated secundum atrial septal defect in children: A longitudinal study. Pediatrics. 2006;118:15601565. DOI: 10.1542/peds.2005-3037 
6. Granton JT, Rabinovitch M. Pulmonary arterial hypertension in congenital heart disease. Cardiol Clin. 2002;20:441-457. PMID: 12371012

7. Goetschmann S, Dibernardo S, Steinmann H, Pavlovic M, Sekarski N, Pfammatter, P. Frequency of severe pulmonary hypertension complicating "isolated" atrial septal defect in infancy. Am J Cardiol. 2008;102:340-342. DOI: 10.1016/j.amjcard.2008.03.061

8. Davidson A, Chandrasekaran K, Guida L, Holsclaw DS Jr. Enhancement of hypoxemia by atrial shunting in cystic fibrosis. Chest. 1990;98:543-545. PMID: 2394134

9. Espiritu JD, Kleinhenz ME. Patent foramen ovale is the most prevalent echocardiographic abnormality in adult cystic fibrosis.
Am J Resp Crit Care Med. 2000;161:A71.

10. Warnes CA, Williams RG, Bashore TM, Child JS, Connolly HM, Dearani JA, et al. ACC/ AHA 2008 guidelines for the management of adults with congenital heart disease: a report of the American College of Cardiology/American Heart Association Task Force on Practice Guidelines (Writing Committee to Develop Guidelines on the Management of Adults With Congenital Heart Disease). Developed in Collaboration With the American Society of Echocardiography, Heart Rhythm Society, International Society for Adult Congenital Heart Disease, Society for Cardiovascular Angiography and Interventions, and Society of Thoracic Surgeons. J Am Coll Cardiol. 2008;52:e143-e263. DOI: 10.1016/j. jacc.2008.10.001

11. Belton M, Gyi KM. Improvement in pulmonary function following closure of a patent foramen ovale in a man with cystic fibrosis. J R Soc Med. 2009;102:S59-S62. DOI: 10.1258/jrsm.2009.s19013

Cite this article as: Saidi $\mathrm{A}$, Carveth $\mathrm{H}$ Tandar A. Improvement in Pulmonary Function After Closure of Atrial Septal Defect in a Patient With Cystic Fibrosis. Structural Heart Disease. 2017;3(6):187191. DOI: https://doi.org/10.12945/j. jshd.2017.022.17 